\title{
Le directeur de projet comme Chief trouble maker
}

Entretien avec Alain P. Michel

\section{Yves Dubreil}

\section{(2) OpenEdition}

\section{Journals}

Édition électronique

URL : http://journals.openedition.org/artefact/6781

DOI : $10.4000 /$ artefact.6781

ISSN : 2606-9245

Éditeur :

Association Artefact. Techniques histoire et sciences humaines, Presses universitaires du Midi

\section{Édition imprimée}

Date de publication : 7 janvier 2021

Pagination : 243-252

ISBN : 978-2-8107-0706-5

ISSN : 2273-0753

\section{Référence électronique}

Yves Dubreil, "Le directeur de projet comme Chief trouble maker », Artefact [En ligne], 13 | 2020, mis en ligne le 23 décembre 2020, consulté le 26 décembre 2020. URL : http://journals.openedition.org/ artefact/6781; DOI : https://doi.org/10.4000/artefact.6781

\section{(c) (i) (2) $\Theta$}

Artefact, Techniques, histoire et sciences humaines est mise à disposition selon les termes de la Licence Creative Commons Attribution - Pas d'Utilisation Commerciale - Pas de Modification 4.0 International. 


\section{Le directeur de projet comme Chief trouble maker}

\section{Entretien avec Alain P. Michel}

\section{Yves Dubreil}

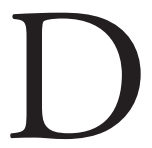

e l'ingénieur Yves Dubreil, on peut dire assurément qu'il a " un losange à la place du cœur ». Entré en 1969 à la Régie Renault, il y a effectué toute sa carrière. Il a déployé la "gestion de projet » en tant que directeur du projet Twingo (1989-1993), puis, à partir de 2003, comme directeur de l'innovation de Renault SAS. Sollicité en 2010 pour devenir président de l'association Renault Histoire, alors qu'il avait fait valoir ses droits à la retraite, il accepte cette proposition, convaincu, dit-il, que «l'histoire est le plus court chemin vers l'innovation".

\section{Ingénieur à vie chez Renault}

\section{Alain P. Michel (APM) : Quelle a été ta formation d'ingénieur ?}

Yves Dubreil (YD) : Je suis né à Rennes en 1947, partageant mon enfance entre la galerie d'art de mon père et une passion pour l'automobile qui se traduisait par de multiples dessins de voitures qui enrichissaient mes cahiers d'écolier. J'ai rencontré Jean-Martin Folz en prépa à Ginette ${ }^{1}$, j'ai préparé les concours avec lui. À notre sortie de Polytechnique, Jean-Martin a d'abord travaillé dans la fonction publique avant de rejoindre le secteur privé et de diriger Peugeot pendant dix ans (1997-2007). De mon côté,

1. Lycée Sainte Geneviève de Versailles.

95 Yves Dubreil, « Le directeur de projet comme Chief trouble maker. Entretien avec Alain P. Michel », Artefact, 13, 2020, p. 243-252. 
j'ai « pantouflé " pour entrer au plus vite dans l'automobile chez Renault où j'ai fait carrière. Considérant qu'un petit dessin vaut mieux qu'un long discours, j'ai continué à émailler les comptes rendus de réunions de dessins humoristiques et autocritiques. C'est pour cela que je me présente souvent comme un « X-autodidacte »! (Fig. 1)

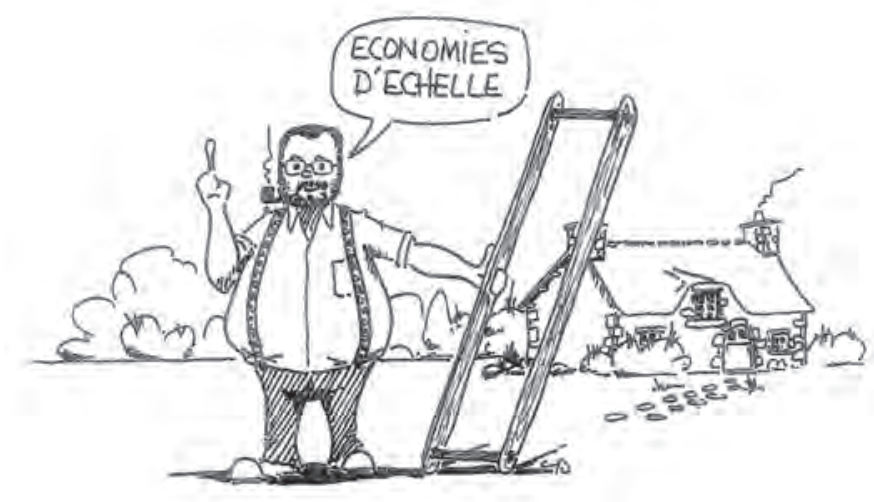

Fig. 1. - Autoportrait d'un Chief Trouble Maker

\section{à bretelles}

La réduction troublante des échelons hiérarchiques pour réduire les délais et le coût d'un projet.

Dessin d'Yves Dubreil, 2013

\section{APM : Quelles ont été les principales étapes de cette carrière d'ingénieur Renault?}

YD : J'ai été pendant cinq ans responsable de l'unité-sièges avant d'accéder enfin à des projets plus directement en lien avec l'automobile, à savoir le concept car Mégane et surtout Twingo. C'est alors que j'ai attrapé le virus de la prospective et de l'innovation. Après un nouveau passage par la case projets (Laguna 2, Velsatis et Espace 4), je suis revenu à l'ingénierie avec la responsabilité d'y organiser le développement de l'innovation. C'est ainsi que j'ai terminé ma carrière comme Chief Trouble Maker, rôle tout droit sorti de la Silicon Valley, que je traduirais par «fouteur de merde en chef ». 
APM : L'innovation en entreprise n'est-elle possible qu'avec des perturbateurs comme toi ?

YD : Je me souviens en particulier d'une réunion qui s'est tenue dans le bureau de Raymond Lévy ${ }^{2}$ avec mes collègues directeurs de projets. Il nous a déclaré : "Dans cette entreprise, 59995 personnes mettent de l'ordre, nous, vous et moi, nous devons mettre du désordre ${ }^{3}$ !»

J'ai eu la chance d'être dans une entreprise nationalisée dans laquelle il y avait une gestion intelligente des cadres supérieurs, une direction des ressources humaines à la recherche d'une diversité féconde, propice à la mise en place de dispositifs flexibles et innovants. C'est ce qui m'a permis d'avoir une vie professionnelle atypique à l'époque ; pas seulement à cause de mon usage immodéré des bretelles colorées et de ma passion de la bonne chère... J'ai connu des périodes de mise au placard qui ont été suivies de rebonds. Je n'ai pas recherché les premières places, mais une place à part.

APM : Qu'est-ce qui a été si particulier, si spécifique dans ton parcours? Mon activité d'ingénieur a été marquée par la transversalité et le travail coopératif dans le développement de l'innovation. Que ce soit à l'unité-sièges où dans l'aventure Twingo, il a été possible, sans avoir besoin d'en demander l'autorisation, de concevoir et mettre en place des organisations, des structures et des outils plus performants, avec l'entière liberté d'y associer des acteurs et compétences extérieurs à l'entreprise.

\section{Relations ingénieurs, cadres et managers dans une grande entreprise}

\section{APM : En quoi ta façon de travailler était-elle différente de celle d'avant?}

YD : Au début du $\mathrm{xx}^{\mathrm{e}}$ siècle, il existait une distinction entre les études de conception des véhicules et les méthodes de fabrication. Le rôle des ingénieurs de conception est resté dominant jusque vers les années 1940. Ensuite, les ingénieurs process ${ }^{4}$ ont été le soutien de la croissance des Trente

2. PDG de la régie Renault de 1986 à 1992.

3. Après la crise de 1983-1984, environ 60000 personnes travaillaient dans l'entreprise Renault.

4. Dont Pierre Bézier est un bel exemple. 
Glorieuses 5 . Puis, au cours des années 1970, on a commencé à se soucier sérieusement des attentes des clients en réalisant des études sociologiques (par exemple la Renault 5, la Renault 16, l'Espace, la Twingo, le Scénic, etc.), il a fallu attaquer des nouveaux marchés pour rentabiliser les tickets d'entrée, en particulier pour l'industrie automobile dont le talon d'Achille était l'intensité capitalistique c'est-à-dire le poids important des investissements indispensables tout en restant compétitif sur le marché.

Or, les systèmes séquentiels coûtent cher. Les cascades de transmissions des dossiers depuis l'ingénierie avancée jusqu'à l'industrialisation et la commercialisation allongent le temps entre la conception d'un véhicule et sa vente. À la suite de la crise des années 1980, la Régie a entrepris une réflexion collective pour définir des chemins efficaces qui permettraient à Renault de sortir du gouffre. Elle s'est organisée autour du MIDES (Mutations industrielles et dynamique économique et sociale $)^{6}$. À partir des années 1990, on a cherché à optimiser tout cela en raccourcissant les délais. Cela a été possible grâce aux "structures projet " qui, en développant le travail coopératif, ont permis de trouver des compromis beaucoup plus efficaces. Cela dit, il a fallu organiser la confrontation entre les différents métiers afin de répondre aux contraintes du projet lui-même, et cela remettait en cause des tas de théories ou de modèles qui font le bonheur des consultants au détriment de l'efficacité. On sait ce qu'il faut faire, mais on ne le fait pas parce que cela choque notre logique de raisonnement. On a eu beaucoup de mal à s'imposer, car comme le dit Jean-René Fourtou ${ }^{7}$ : «les folles raisons du projet valent mieux que les bonnes raisons du budget ».

\section{Une expérience unique de gestion d'un projet innovant}

\section{APM : Comment s'est passée concrètement chez Renault la mise en place de la " gestion par projet"?}

YD : Le soutien du P.-D.G. a été déterminant pour remporter les « bras de fer " avec les bureaucrates qui prospèrent dans les grandes organisations.

5. Voir l'article d'Alain P. Michel dans ce numéro.

6. Monnet, 2013.

7. PDG de Rhône-Poulenc puis de Vivendi. 
Le 5 janvier 1989 à 17 h 00, je suis convoqué par le président Lévy qui me dit d'emblée :

Monsieur Dubreil, vous entrez dans ce bureau comme directeur aux achats, vous en sortirez directeur du " projet X06 ». Je n'admets pas que vous refusiez! Vous avez un projet qui a du talent et des originalités, mais qui est loin d'être rentable. Vous avez trois mois pour nous démontrer qu'il peut l'être. Évidemment, si vous pouvez nous convaincre que c'est possible, vous serez chargé de le mener à son terme dans ces conditions. Sinon on l'abandonnera, non sans avoir expliqué les raisons de cette impossibilité !

En quelques minutes, ma vie venait de changer. Pourquoi moi ?... Il se trouvait que je venais d'être chargé d'un petit projet : le concept car "Mégane " que la Régie prévoyait de présenter au salon de Francfort de 1989. Or j'avais proposé et réussi à le terminer un an plus tôt, en octobre 1988 à Paris, l'année où le salon est devenu le "Mondial de l'automobile ». On m'a dit que c'est grâce à cette réduction de délai de plus de $50 \%$ que j’ai été retenu pour le " projet X06 » apparemment impossible.

\section{APM : Quel était ce " projet X06» ?}

YD : Depuis le milieu des années 1970, Renault s'interrogeait sur la possibilité de faire un deuxième véhicule de bas de gamme, à côté de la lignée R 5 , son nom de code était le VBG (véhicule de bas de gamme) plus innovant, plus décalé. Le 11 novembre 1977 après-midi, au cours d'une réunion qui s'est tenue dans les salons panoramiques du Sofitel-Sèvres, le projet VBG a été abandonné. Les maquettes de style ne créaient pas d'attrait, et surtout ce n'était pas rentable face à une $\mathrm{R} 5$ phase 2 très compétitive qui était annoncée moins de deux ans après. Cependant des projets ont été testés et finalement un projet de monocorps sympathique, bien amélioré par le design de Patrick Le Quément, a été présenté début décembre 1988 à la direction générale ${ }^{8}$. L'histoire raconte qu'après la présentation du dossier (toujours pas rentable, et il s'en faut de $20 \%$ au moins...), la maquette (très proche du dessin final de Twingo 1) est montrée et fait sourire le président et donc toute la DG... Ils décident de donner une dernière chance au projet en nommant un directeur de projet. C'était l'objet de ma convocation par Lévy, le 5 janvier 1989. C'est une sorte de rite qui me donnait le

8. Bellu, 2010. 
pouvoir de tout tenter pour proposer des solutions capables de rentabiliser ce projet. J'ai alors utilisé deux dessins pour passer mon message à l'équipe projet : en particulier celui du tunnel pour montrer comment mettre en œuvre le design to cost (Fig. 2).

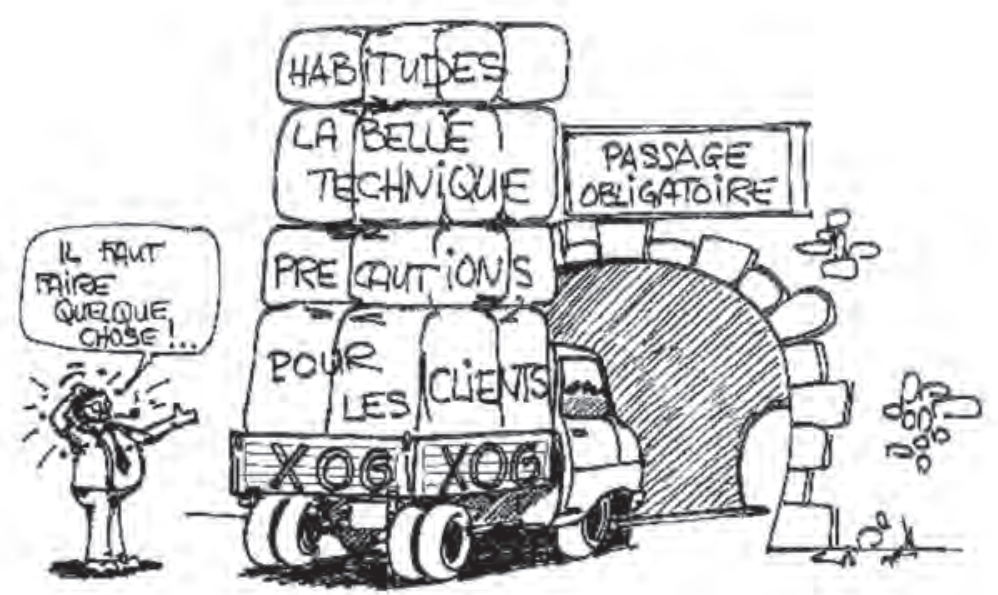

Fig. 2. - « Devant le tunnel»

Exemple de dessin utilisé par Yves Dubreil pour passer à l'équipe projet le message sur le design to cost, incontournable pour réussir la Twingo.

Dessin d'Yves Dubreil, 1989

\section{La mise en place progressive des règles de la gestion par projet}

\section{APM : Comment l'équipe X06 a-t-elle travaillé ?}

YD : Nous avons tout de suite été charmés par l'objet. Dans mon cas, dès le lendemain de ma rencontre avec Lévy, j'ai couru sur les pistes d'essai de Renault à Aubevoye (Eure) pour conduire et surtout regarder rouler la maquette roulante de la future Twingo. J'ai été séduit car c'est un design qui " bouge bien ", qui est dynamique avec ses roues aux quatre coins et sa bouille sympa. Nous nous sommes mis au travail avec enthousiasme car c'était l'occasion d'une relance inespérée de notre bébé : la gestion de projet. Face à ce produit innovant, nous avons forgé au fur et à mesure les outils du management pour sept raisons au moins : 
1. Le design to cost. Le coût est la contrainte absolue et non pas, comme c'est souvent le cas, la simple résultante des contraintes techniques, industrielles et commerciales. On a fait venir nos trois fournisseurs et on leur a dit : " pour cette pièce du X06, toutes les estimations donnent des chiffrages autour de 250-255 francs ". J'ai sorti un billet de 200 francs de ma poche et dit : « la seule réponse qui m'intéresse, c'est qu'est-ce que vous pouvez me faire pour 200 francs. Tout le reste est négociable ». Et ça a marché !

2. La confrontation du coût de chaque fonction. On a mis en place un système très simple (tenant sur un simple tableau Excel, je crois) qui, pour chaque fonction du projet, donnait, sur deux colonnes, d'une part l'objectif de coût et de l'autre le coût probable, résultant du coût prévu corrigé par les risques et opportunités probabilisés. La gestion du projet consistait à trouver des solutions pour que le coût probable reste inférieur à l'objectif de coût. Chaque semaine, chaque groupe fonction faisait son reporting, de façon à ce que l'équipe projet puisse prioriser ses actions sur les fonctions qui avaient le plus de mal.

3. Tout problème se règle in situ. On a réussi à mettre tous les acteurs du projet sur un plateau commun, en regroupant tous les métiers impliqués dans chaque fonction. Les réticences étaient énormes. Le soutien de la direction générale a été déterminant pour convaincre les récalcitrants. On a aussi multiplié les rencontres sur le terrain (usines, fournisseurs, réseau commercial, etc.) avec les acteurs locaux.

4. Un appui sur la recherche en sciences sociales. Au début des années 1990, j'ai sollicité le soutien éclairé de Christophe Midler du Centre de recherche en gestion (CRG) de l'École polytechnique?. Je l'avais déjà rencontré, en particulier dans l'expérience du MIDES. L'objectif était qu'il vive l'expérience avec nous pour observer et participer à nos décisions. Il a accepté, malgré la contrainte de ne rien publier avant la sortie de la Twingo sur le marché en 1993. Il a été largement récompensé par la publication en 1993 de son best-seller, L'auto qui n'existait pas ${ }^{10}$. C'est le premier des projets innovants de bas de gamme avant "l'épopée Logan ${ }^{11}$ " en 2004, puis, en 2015, l'idée "d'innover à l'envers " pour la conception frugale du modèle Kwid, destiné initialement au marché indien ${ }^{12}$. Ces trois projets partagent la pratique d'une " ingénierie concourante ", c'est-à-dire la mobilisation de tous les métiers du début à la fin, en vue de faire marcher le plus longtemps possible la machine

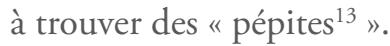

9. Perriaux, 1998.

10. Midler, 2004.

11. Midler et al., 2012.

12. Midler et al., 2017.

13. Dubreil, 2017. 
5. Une internationalisation des bonnes pratiques avec le Club de Montréal. Sur les conseils de Gérard Dubrulle (de la DRH), nous avons lancé une learning expedition à Montréal et créé un club destiné à regrouper des responsables de projets innovants et atypiques pour échanger sur nos bonnes (et mauvaises) pratiques ${ }^{14}$. Parmi les " métarègles " de ce club, l'absence de structure et de hiérarchie : nous étions tous des présidents ! Pas de budget : chacun se débrouillait avec son entreprise ou ses ressources propres. La notoriété du Club de Montréal a été telle qu'un des patrons de Renault a été surpris d'être questionné sur son existence, sa nature et sa raison d'être lors des contacts avec des directions générales de nos concurrents!

6. Le respect des délais comme priorité. La réduction des délais est un enjeu énorme car c'est le carburant du progrès. Quelques mois avant la sortie de la Twingo en série, on a constaté que le support de pompe à eau ne tenait pas en endurance. Il fallait une solution... et très vite ! Par une mobilisation générale avec les processus existants, cela risquait de se traduire par un retard de deux à trois mois sur un chemin critique du projet... On a convoqué dare-dare trois fournisseurs de ce type de pièces et on leur a fait partager la même salle d'attente. On a d'abord demandé à celui qui nous paraissait le plus réactif : " par le processus classique, on perd deux à trois mois. Pouvez-vous d'ici la fin du week-end prochain nous proposer une solution temporaire, sans doute plus chère, mais qui nous permette de valider très vite cette solution de démarrage ? " Il demande à téléphoner à son patron sur-le-champ. Quelques minutes après nous avons sa réponse : " on peut vous promettre pour avant dimanche soir une dizaine de pièces sur une définition certes plus coûteuse mais permettant d'assurer la montée en cadence. » Bingo ! Tout cela a été obtenu en mettant toutes les compétences, les bonnes volontés et la capacité de décision autour d'une même table. Moi, je n'apportais que l'autorisation de ne pas respecter l'objectif de coût, mais le jeu en valait la chandelle !

7. Pas de vaches sacrées pour une vache à lait ! Pour obtenir du marketing la réduction des frais de commercialisation, j'ai dû les convaincre en menaçant de vendre Twingo dans les supermarchés. A posteriori, tous les commerçants m'ont confirmé que la vente de la Twingo était une vache à lait pour le réseau : elle se vendait toute seule ! Il faut dire que sa version unique était aussi bien équipée que les versions moyennes de la concurrence : "Une voiture tout compris, équipements et remises inclus!». La gestion de projet démontre que l'innovation peut être frugale et venir d'en bas, au contraire de ces innovations entropiques c'est-à-dire conçues par des bureaux d'études déconnectées de la réalité qui ne s'intéressent qu'au haut de gamme et font exploser les contenus, la complexité et les coûts ${ }^{15}$.

14. Dubreil et al., 2004.

15. Dubreil, 2017. 


\section{Conclusion : « La prospective se nourrit de la rétrospective"}

\section{APM : Que dirais-tu pour conclure ? Quel regard rétrospectif ?}

YD : Grâce à des travaux sur les projets, on a découvert qu'il y avait une très forte corrélation entre les projets loupés et l'absence de confrontation des différents métiers de l'entreprise. Une bonne organisation projet est donc celle qui organise ces confrontations entre tous les acteurs, non pas par une série chronologique de passage de relais - comme le préconisaient les règles de la " qualité totale »- mais bel et bien en mettant dès le départ toutes les compétences à contribution dans une confrontation féconde.

Critère plus important encore, une étude socio-psycho réalisée sur le programme M2-S de Renault a montré que les modifications de culture et de comportement dans une entreprise ne se font pas par l'oukase d'un patron ou d'un consultant, mais en confrontant les acteurs internes qui partagent une culture ancienne avec les contraintes nouvelles imposées par les marchés, les règlements, la finance, etc.

À ce titre, le fait que Louis Schweitzer, successeur de Raymond Lévy en 1992, se soit déclaré opposé au projet Twingo tant qu’il n'était pas convaincu de sa bonne rentabilité, nous a contraints à être performants. Comme le disait François Andrieux, " on ne s'appuie bien que sur ce qui résiste ${ }^{16}$ !"

Lors de mon départ à la retraite, j'ai découvert au sein de Renault Histoire la gouvernance des bénévoles. C'était un autre monde où le seul pouvoir dont on dispose est celui de l'exemplarité afin de partager avec d'autres l'envie de participer à une aventure féconde ! Pour moi, la boucle est bouclée et le travail sur la culture de Renault, construite par son histoire, est le ferment le plus fort d'une prospective féconde sur l'automobile et la mobilité. La prospective se nourrit de la rétrospective!

16. Fameuse réplique de François Andrieux (1759-1833) au consul Bonaparte lors d'une rencontre en 1802 à l'Institut de la section mécanique. Il devient ensuite professeur de lettres à Polytechnique et membre de l'Académie française. 
Yves Dubreil

\section{Bibliographie}

Bellu Serge, De Simca à Renault. 40 ans de design sur les pas de Patrick Le Quément, ETAI, Boulogne-Billancourt, 2010.

Dubreil Yves, Jolivet François et Navarre Christian, « Le club de Montréal a dit... une conspiration pour la gestion par projets ", Le journal de l'école de Paris du management, 46, 2004/2, p. 15-22.

Dubreil Yves, «Fiche de lecture sur Innover à l'envers de Midler, Jullien \& Lung », note du 15 mars 2017, reprise dans Renault Histoire, n 46, octobre 2019, p. 192.

Midler Christophe, L'Auto qui n'existait pas. Management des projets et transformation de l'entreprise, Dunod, Paris, 2004 (rééd. 2012).

Midler Christophe, Jullien Bernard et Lung Yannick, L'Épopée Logan. Nouvelles trajectoires pour l'innovation, Dunod, Paris, 2012.

Midler Christophe, Jullien Bernard et Lung Yannick, Innover à l'envers. Repenser l'innovation et la conception dans un monde frugal, Dunod, Paris, 2017.

Monnet Jean-Claude, Un sociologue chez Renault. De Pierre Bourdieu à Carlos Ghosn (1984-2005), Armand Colin, Paris, 2013.

Perriaux Anne-Sophie, Renault et les sciences sociales, 1948-1991, Seli Arslan, Paris, 1998. 